\title{
Is the patch size distribution of vegetation a suitable
} indicator of desertification processes?:

7 Kéfi, S. ${ }^{1 *}$, Alados, C.L. ${ }^{2}$, Chaves, R.C.G. ${ }^{3}$, Pueyo, Y. ${ }^{4}$, and Rietkerk, M. ${ }^{5}$

$9 \quad{ }^{1}$ Department of Biology, Darmstadt University of Technology. Schnittspahnstr. 10. 64287

10 Darmstadt, Germany

$11{ }^{2}$ Instituto Pirenaico de Ecología (CSIC). Avda. Montañana 1005. P.O.Box. 202. 50080

12 Zaragoza, Spain.

13 Max-Planck-Institut für Kernphysik, P.O. Box 103980, D 69029 Heidelberg, Germany

$14{ }^{4}$ Geography Department, University of Zaragoza, Campus Plaza San Francisco. Pedro

15 Cerbuna, 12. 50009 Zaragoza, Spain

$16{ }^{5}$ Department of Environmental Sciences, Copernicus Institute, Utrecht University, P.O. Box

1780115,3508 TC Utrecht, The Netherlands

18

$19 *$ Corresponding author. E-mail: kefi@bio.tu-darmstadt.de

20

21 Manuscript type: Comment 
22 With ongoing climate change, the search for indicators of imminent ecosystem shifts is

23 attracting increasing attention (e.g. Scheffer et al. 2009). Recently, the spatial organization of

24 ecosystems has been suggested as a good candidate for such an indicator in spatially

25 structured ecosystems (Rietkerk et al. 2004, Kéfi et al. 2007a, Guttal and Jayaprakash 2009).

26 Arid ecosystems are well-known for the spatial organization of their vegetation cover, which

27 is often characterized by clumps of vegetation in an otherwise bare soil matrix. Two recent

28 studies revealed that the distribution of the vegetation patch size can be described by a power

29 law over a wide range of environmental conditions in arid ecosystems (Kéfi et al. 2007a,

30 Scanlon et al. 2007). Furthermore, deviations from power laws were observed under high

31 grazing pressures, leading to the hypothesis that such deviations could be used as indicators of

32 approaching desertification in arid ecosystems (Kéfi et al. 2007a). This hypothesis now needs

33 to be validated with additional field data, before it can be confidently used as a tool to monitor

34 degradation in arid ecosystems.

36 In a recent study, Maestre and Escudero (2009) (hereafter referred to as ME09) aimed to test

37 this hypothesis with data from 29 steppes located on a rainfall gradient in southeast Spain. In

38 all of their sites, the patch size distribution was best described by a truncated power law

39 (TPL) ${ }^{1}$. Relating the scaling exponents of these TPLs to soil variables, the authors concluded

40 that 1) the patch size distribution was not directly related to desertification but rather that 2)

41 vegetation cover could be used to monitor desertification. We argue in this comment that the

42 analyses of ME09 do not allow them to draw these conclusions, for the following two

43 reasons. First, because all of their sites were characterized by TPLs, the authors looked only

1 We use here the same terminology as in Kéfi et al. (2007) and Maestre and Escudero (2009), where a TPL refers to a power law with exponential cutoff, i.e. such that $\mathrm{N}(\mathrm{S})=\mathrm{CS}^{-\gamma} \exp \left(-\mathrm{S} / \mathrm{S}_{\mathrm{c}}\right)$ with $\mathrm{N}$ the number of patches of size $\mathrm{S}, \mathrm{C}$ a constant, $\mathrm{Y}$ the scaling exponent (positive) and $\mathrm{S}_{\mathrm{c}}$ the patch size above which $\mathrm{N}$ decreases faster than in a power law (positive). 
44 at the scaling exponents $\gamma$ of these TPLs to compare the degradation level of the sites.

45 However, $\gamma$ was not proven to vary with degradation in a consistent manner, and therefore

46 the analyses of ME09 do not allow them to conclude whether vegetation cover is better

47 related to degradation than patch size distribution. Second, although the vegetation cover is

48 often a simple and efficient indicator of degradation, the authors do not take into account the

49 increasing amount of literature that strongly suggests vegetation cover in arid ecosystems is

50 likely to respond in a discontinuous way to gradual, external changes (Rietkerk et al. 1996,

51 Lejeune et al. 1999, Scheffer et al. 2001, von Hardenberg et al. 2001, Kéfi et al. 2007b). In

52 these cases, the vegetation cover alone simply does not provide information on the actual

53 distance to desertification.

55 The categorization proposed by Kéfi et al. (2007a) is a qualitative one in that it does not 56 provide a quantifiable distance to extinction: a shift (in time) from a pure power law to a TPL 57 suggests that an ecosystem is degrading and approaching the desertification threshold. The 58 sites studied by ME09 are all described by TPLs. Among sites characterized by similar patch 59 size distributions, Kéfi et al. (2007a) do not propose any criteria to distinguish among sites of 60 varying degradation; currently, such criteria are sorely lacking. In an attempt to compare the 61 degradation levels of their 29 sites, ME09 investigated changes in the scaling exponent $\gamma$ of 62 the TPL among the different sites. This was not part of the hypothesis formulated by Kéfi et 63 al. (2007a). It is an interesting approach, but it implicitly assumes that $\gamma$ varies consistently 64 with the level of stress, which has not been proven to be the case. In fact, in the data analyzed 65 by Kéfi et al. (2007a), there does not appear to be any consistent variation of $\gamma$ among sites 66 characterized by different stress levels (i.e. grazing pressures). For example, with increasing 67 grazing pressure (from medium to high) the absolute value $\gamma$ of the TPL decreases in the data 
68 from Spain but increases in the data from Morocco and Greece (see Fig. 1 in Kéfi et al.

69 (2007a)). The lack of a clear relationship between $\gamma$ and the stress level could very well

70 explain why ME09 find that $\gamma$ is not related to the perennial cover. It is noteworthy that this

71 result is in agreement with previous studies on steppes dominated by Stipa tenacissima in the

72 arid Mediterranean region. For example, it has been shown that the spatial distribution of $S$.

73 tenaccisima is not clearly related with its abundance (see Table 1 in Alados et al. (2006)).

74 Furthermore, the exponent $\gamma$ alone does not provide a complete description of the shape of

75 the TPL; the location of the cutoff, $S_{c}$, cannot be ignored. Indeed, the latter describes where

76 the deviation from power law behavior begins, and it is this deviation which was proposed to

77 be linked to the level of degradation in Kéfi et al. (2007a). Thus, we doubt whether $\gamma$ is the

78 correct parameter to investigate. Further theoretical and empirical work is needed in order to

79 identify the parameters which are best correlated to the stress level and which therefore

80 should be monitored.

82 Another concern regarding the analysis of ME09 is that, when fitting TPLs to their data, they

83 find negative power law exponents in the vast majority of their sites (22/29 sites listed in

84 ME09 Table 1 and 7/8 sites illustrated in ME09 Fig. D1), in stark contrast to the positive

85 power law exponents observed by Kéfi et al. (2007a). A TPL with a negative power law

86 exponent can be understood as follows: the number of patches $\mathrm{N}(\mathrm{S})$ actually increases with

87 size $S$ until some intermediate path size is reached, at which point $N(S)$ begins to decrease.

88 Thus, in ME09's distributions, it is common for smaller patches to be less abundant than

89 patches of intermediate size. For this reason, a TPL does not appear to be the most appropriate

90 model to use to fit the data. The distributions found by ME09 actually suggest the presence of

91 a dominant spatial scale, contrary to the scale invariance observed by Kefi et al. (2007a). 
92 Indeed, some arid areas are characterized by regular vegetation patterns (Rietkerk and van de

93 Koppel 2008), where patch size distributions do not follow power laws but instead reflect a

94 characteristic patch size (or a range of patch sizes). Manor and Shnerb (2008) developed a

95 promising model which can reproduce both the irregular patterns described by power law

96 distributions and the regular patterns characterized by a dominant spatial scale. They showed

97 how the relative strength of competition and facilitation can drive the type of pattern that

98 emerges; strong facilitation favors irregular pattern formation while strong competition favors

99 regular patterns. In systems characterized by regular patterns, it has been suggested that the

100 shape of the patterns can be used to gauge the level of degradation, with spot patterns being

101 the last to occur before desertification (Rietkerk et al. 2004). Further research is needed to

102 determine if these findings can indeed be applied to the sites studies by ME09. More

103 generally, what is currently lacking is a robust way of characterizing the spatial organization

104 of ecosystems, since, depending on the type of patterns (which emerge from different

105 underlying ecological mechanisms), the indicators that need to be monitored may vary.

107 Before patch size distributions can be used as a monitoring tool in systems characterized by 108 irregular patterns, many technical issues need to be addressed and further tests need to be 109 conducted in the field. From a practical point-of-view, the patch size distribution is indeed a 110 more complicated tool than the vegetation cover. Among others, there are issues with the 111 binning of the data and the fitting of the mathematical functions.

112 Traditionally, data is binned when visualizing frequency distributions (Newman 2006, Bauke 113 2007, White et al. 2008, Clauset et al. In press). When the data are binned into bins of equal 114 sizes (so-called linear binning), the right-hand side of the distribution is often noisy: the 115 largest elements are rare, and, therefore, each bin contains only a few elements which creates 
116 large variations in bin counts among bins (Newman 2005, Bauke 2007). This is a concern

117 when dealing with patch size distributions, since we are especially interested in the behavior

118 of the putative power law in the area around the largest, i.e. the rarest, patches. To decrease

119 the noise in the right-hand tail of the distribution, logarithmic binning is typically employed,

120 where the bins in the tail of distribution receive more elements than with linear binning.

121 Various techniques have been proposed to estimate the optimum bin size (e.g. Sturges' rule,

122 Scott's rule, and the Freedman-Diaconis rule); all strive to achieve a reasonable balance

123 between the number of bins and the number of elements in each bin. However, these

124 techniques do not always yield consistent results, which makes the choice of binning fairly

125 arbitrary. A better way of plotting the data is to use the cumulative distribution function,

126 which does not involve the binning of the data (Newman 2005, Bauke 2007, White et al.

127 2008).

128 After binning the data, a linear fitting of the log-log transformed data is typically performed

129 using least squares regression (Newman 2005, Bauke 2007, White et al. 2008, Clauset et al.

130 In press). Fitting methods based on binning and least squares regression are widely used in

131 ecology and in other fields to fit models to data and to estimate the scaling exponents of

132 frequency distributions. White et al. (2008) recently demonstrated that such methods give

133 biased results and therefore cannot be relied upon. While these biases are dangerous with

134 regards to estimating the scaling exponent of a distribution, binned-based methods can also

135 lead to differences in the determination of which distribution best fits the data. For example, a

136 data set that is best described by a power law using a given bin size could be best described

137 by a TPL when using a different bin size.

138 Independently of the way the data are plotted, a reliable alternative to least square linear

139 regression is to use fitting methods based on maximum likelihood estimation (MLE) to 
140 extract the scaling exponent of the frequency distribution (e.g. Goldstein et al. 2004). White et

141 al. (2008) showed that MLE is the single most accurate method for estimating the scaling

142 exponents of frequency distributions. Currently, MLE is available for the pure power law

143 distribution (Goldstein et al. 2004, Newmann 2005, Bauke 2007) but not for the TPL

144 distribution as defined here, which limits the application of MLE to this particular case for

145 now, but is a promising line of future research.

147 In conclusion, although looking at the vegetation cover is still the most obvious and practical

148 way of assessing the "health" of an arid ecosystem, there are notable cases where the cover

149 fails to predict desertification. Desertification can occur in sudden shifts, where ecosystems

150 switch from an unknown vegetation cover to desert. Theoretical studies increasingly suggest

151 that ecosystems which include facilitation may respond to gradual external changes in an

152 abrupt, rather than gradual manner (e.g. Lejeune et al. 1999, Scheffer et al. 2001, von

153 Hardenberg et al. 2001, Rietkerk et al. 2004, Kéfi et al. 2007b). In these cases, the vegetation

154 cover is not an appropriate indicator of proximity to shifts and, therefore, other potential

155 indicators (e.g. the patch size distribution) need to be further developed so that they can be

156 used in addition to the cover. In the meantime, we advocate the continued but cautious use of

157 the cover as a means to gauge an arid ecosystem's health, but reiterate the need to explore

158 more robust techniques before a reliable early-warning system can be implemented. 
Alados, C. L., P. Gotor, P. Ballester, D. Navas, J. M. Escós, T. Navarro, and B. Cabezudo. 2006. Association between competition and facilitation processes and vegetation spatial patterns in alpha steppes. Biological Journal of the Linnean Society 87:103113.

166

Bauke, H. 2007. Parameter estimation for power-law distributions by maximum likelihood methods. The European Physical Journal B - Condensed Matter and Complex Systems 58(2):167-173.

Clauset, A., C. R. Shalizi, and M. E. J. Newman. Power-law distributions in empirical data. SIAM Review. In press arXiv:0706.1062.

deMenocal, P., J. Ortiz, T. Guilderson, J. Adkins, M. Sarnthein, L. Baker, and M. Yarusinsky. 2000. Abrupt onset and termination of the African Humid Period: rapid climate responses to gradual insolation forcing. Quaternary Science reviews 19:347-361.

Foley, J. A., M. T. Coe, M. Scheffer, and G. Wang. 2003. Regime shifts in the Sahara and Sahel: interactions between ecological and climatic systems in northern Africa. Ecosystems 6:524-539.

Goldstein, M. L., S. A. Morris, and G. G. Yen. 2004. Problems with fitting to the power-law distribution. The European Physical Journal B - Condensed Matter and Complex Systems 41(2):255-258.

Guttal, V., and C. Jayaprakash. 2009. Spatial variance and spatial skewness: leading indicators of regime shifts in spatial ecological systems. Theoretical Ecology 2:3-12. 
Kéfi, S., M. Rietkerk, C. L. Alados, Y. Pueyo, V. P. Papanastasis, A. ElAich, and P. C. de Ruiter. 2007a. Spatial vegetation patterns and imminent desertification in Mediterranean arid ecosystems. Nature 449:213-217.

Kéfi, S., M. Rietkerk, M. van Baalen, and M. Loreau. 2007b. Local facilitation, bistability and transitions in arid ecosystems. Theoretical Population Biology 71:367-379.

Lejeune, O., P. Couteron, and R. Lefever. 1999. Short range co-operativity competing with long range inhibition explains vegetation patterns. Acta Oecologica 20:171-183.

Maestre, F. T., and A. Escudero. 2009. Is the patch size distribution of vegetation a suitable indicator of desertification processes? Ecology 90:1729-1735.

Manor, A., and Shnerb, N. 2008. Facilitation, competition, and vegetation patchiness: From scale free distribution to patterns. Journal of Theoretical Biology 253:838-842.

Newman, M. E. J. 2005. Power laws, Pareto distributions and Zipf's law. Contemporary Physics 46(5):323 - 351 .

Rietkerk, M., S. C. Dekker, P. C. de Ruiter, and J. van de Koppel. 2004. Self-organized patchiness and catastrophic shifts in ecosystems. Science 305:1926-1929.

Rietkerk, M., P. Ketner, L. Stroosnijder, and H. H. T. Prins. 1996. Sahelian rangeland development: a catastrophe? Journal of Range Management 49:512-519.

Rietkerk, M., van de Koppel, J. 2008. Regular pattern formation in real ecosystems. Trends in Ecology and Evolution 23(3): 169-175.

Scanlon, T. M., K. K. Caylor, S. A. Levin, and I. Rodriguez-Iturbe. 2007. Positive feedbacks promote power-law clustering of Kalahari vegetation. Nature 449:209-U204.

Scheffer, M., S. Carpenter, J. A. Foley, C. Folke, and B. Walker. 2001. Catastrophic shifts in ecosystems. Nature 413:591-596. 
205 Scheffer M., J. Bascompte, W.A. Brock, V. Brovkin, S.R. Carpenter, V. Dakos, H. Held, E.H. van Nes, M. Rietkerk, and G. Sugihara. 2009. Early-warning signals for critical transitions. Nature 461: 53-59.

208 von Hardenberg, J., E. Meron, M. Shachak, and Y. Zarmi. 2001. Diversity of vegetation patterns and desertification. Physical Review Letters 8719.

210 White, E. P., B. J. Enquist, and J. L. Green. 2008. On estimating the exponent of power-law 211 frequency distributions. Ecology 89:905-912. 